\title{
AGRESIONES SEXUALES COMETIDAS POR DESCONOCIDOS EN ESPANAA: ALGUNAS CARACTERÍSTICAS DEL AGRESOR Y ALGUNOS ASPECTOS INTERACTIVOS DEL CRIMEN
}

\author{
Heriberto Janosch González \\ Máster en Investigación en Psicología, \\ Universidad Nacional de Educación a Distancia. \\ Doctorando, Universidad Camilo José Cela
}

Juan Enrique Soto Castro

Dr. en Psicología, Universidad Camilo José Cela

Jefe de la Sección de Análisis de la Conducta del Cuerpo Nacional de Policía

Resumen: Propósito: En este estudio, a partir de una muestra de agresiones sexuales cometidas en España contra mujeres mayores de 12 años, analizamos variables referentes a las características del agresor sexual, al ambiente, a la víctima, y a la posible reacción de terceros; y las vinculamos con cuatro dimensiones de temáticas conductuales del agresor en la escena del crimen: Hostilidad, Control, Criminalidad e Intimidad.

Método: 85 casos de agresiones sexuales en España fueron asignados a cada una de las cuatro temáticas conductuales. Posteriormente se identificaron 10 variables referentes a las características del agresor y del ambiente, y a conductas de la víctima y de testigos del hecho. Con los valores de las diez variables y los valores de asignación a las cuatro temáticas, se realizó un escalamiento multidimensional (MDS) para observar cómo se asociaban entre ellas. Por último, se estudió las edades de los agresores en cada uno de los cuatro temas.

Resultados: No se encontraron diferencias significativas respecto a las edades en cada grupo temático. La agresión en lugares públicos, la resistencia de la víctima gritando, y la concurrencia de terceras personas 
brindando ayuda, se asociaron al tema de Hostilidad. La agresión en la zona privada de la víctima y problemas psiquiátricos, están asociados a los agresores asignados al tema de Control. Aquellos agresores sexuales asignados a la temática de Criminalidad suelen actuar en la zona privada de la mujer, y tener problemas de adicción a drogas. Por último, los agresores del tema Intimidad actúan indistintamente en la zona privada de la víctima o en la suya propia, tienen pareja estable, y problemas con el alcohol. En este caso la víctima suele escapar de la agresión.

Conclusión: Hemos encontrado algunas asociaciones con variables referidas a características del agresor, del ambiente, a conductas de la víctima y de terceras personas que son testigos del hecho; y las cuatro temáticas conductuales. Estos conocimientos podrían ser de utilidad a la hora de elaborar perfiles psicológicos hipotéticos del agresor en casos futuros. La interacción del delincuente con la víctima, testigos y ambiente se muestra crucial, y esperamos que los futuros estudios se centren más en el evento criminal en su totalidad, y no exclusivamente en las conductas del agresor en la escena del crimen.

Palabras clave: Perfilación criminológica, Agresiones sexuales, España, Análisis de la conducta, Investigación policial

Abstract: Purpose: In this study, based on a sample of sexual assaults committed in Spain against women over 12 years of age, we analyze variables related to the characteristics of the sexual aggressor, the environment, the victim, and the possible reaction of third parties; and we link them with four dimensions of behavioral issues of the aggressor at the crime scene: Hostility, Control, Crime and Intimacy.

Method: 85 cases of sexual assault in Spain were assigned to each of the four behavioral themes. Subsequently, 10 variables were identified concerning the characteristics of the aggressor and the environment, and to the victim's behaviors and witnesses of the event. With the values of the ten variables and the values assigned to the four themes, a multidimensional scaling (MDS) was carried out to observe how they were associated with each other. Finally, we studied the ages of the aggressors in each of the four subjects.

Results: No significant differences were found regarding the ages in each thematic group. The aggression in public places, the resistance of the screaming victim, and the concurrence of third parties providing help, were associated with the issue of Hostility. The aggression in the private area of the victim and psychiatric problems are associated with the aggressors assigned to the topic of Control. Those sex offenders assigned to the subject of Criminality usually act in the private area of women, and have problems of drug addiction. Finally, the aggressors of the Intimacy issue act indiscriminately in the victim's private zone or in their own private zone, have a stable partner, and problems with alcohol. In this case, the victim usually escapes aggression. 
Conclusion: We have found some associations with variables referring to characteristics of the aggressor, the environment, behavior of the victim and third parties who are witnesses of the event; and the four behavioral issues. This knowledge could be useful when developing hypothetical psychological profiles of the aggressor, in future cases. The interaction of the offender with the victim, witnesses and environment is crucial, and we hope that future studies will focus more on the criminal event as a whole, and not exclusively on the behavior of the aggressor at the crime scene.

Keywords: Criminal profiling, Sexual aggressions, Spain, Behavior analysis, Police investigation

\section{Introducción}

\section{Conductas en la escena del crimen y características del delincuente}

La perfilación criminológica (PC), también conocida en inglés como Criminal profiling, Offender profiling, o simplemente Profiling, tiene como objeto elaborar un perfil psicológico y demográfico de la clase de persona que ha cometido un delito grave, en base a sus conductas deducidas de la escena del crimen (Douglas, Ressler, Burgess \& Hartman, 1986; Hicks \& Sales, 2009; Konvalina-Simas, Turvey \& Kennedy, 2016; Soto CASTRO, 2017, TuRvey, 2012). CANTER y sus colaboradores simbolizan este proceso con la relación $« \mathrm{~A} \Rightarrow \mathrm{C}$ », donde $\mathrm{A}$ son las acciones cometidas por el delincuente durante el crimen y $\mathrm{C}$ las características del mismo (CANTER \& YOUNGS, 2009). El principal fundamento de la PC es el «principio» de Homología: aquellos delincuentes cuyas conductas en la escena del crimen son similares, también serán similares en sus características psicológicas y demográficas, tales como su edad en el momento del crimen, nivel de educación, situación laboral, estado civil, antecedentes policiales o judiciales por delitos de agresión sexual u otros delitos, etcétera. Este "principio» presupone el de la Consistencia Conductual: cada delincuente es consistente en sus conductas criminales, en su modus operandi, a lo largo del tiempo. El «principio» de Homología todavía no ha recibido confirmación, existiendo estudios que lo ponen en duda, como el de MOKROS Y ALISON (2002); aunque también hay estudios que indicarían su validez bajo ciertos supuestos, como tener en cuenta el proceso de toma de decisiones del delincuente durante las fases del crimen (GoodwILL, LEHMANN, BEAUREGARD \& ANDREI, 2016). En su parte práctica, un perfil criminológico (psicológico y demográfico) de un caso en particular es un conjunto de hipótesis acerca de la clase de persona que cometió el delito, y que se entrega, junto a una lista de sugerencias operativas para 
la verificación de tales hipótesis, al equipo de investigación policial (Soto Castro, González Ordi y Pérez Nieto, 2014; Soto Castro, 2017).

\section{Definición de Agresión Sexual}

La definición de agresión sexual que utilizamos para este estudio está tomada directamente de los artículos correspondientes del Código Penal español vigente. El artículo 178 expresa que «[e]l que atentare contra la libertad sexual de otra persona, utilizando violencia o intimidación, será castigado como responsable de agresión sexual con la pena de prisión de uno a cinco años». Por otro lado, el artículo 179 estipula que «cuando la agresión sexual consista en acceso carnal por vía vaginal, anal o bucal, o introducción de miembros corporales u objetos por alguna de las dos primeras vías, el responsable será castigado como reo de violación con la pena de prisión de seis a doce años». Por último, el artículo 180 se refiere a las circunstancias agravantes del delito de agresión sexual, como por ejemplo, «cuando la violencia o intimidación ejercidas revistan un carácter particularmente degradante o vejatorio [...] Cuando la víctima sea especialmente vulnerable, por razón de su edad, enfermedad, discapacidad o situación [...] Cuando el autor haga uso de armas u otros medios igualmente peligrosos, susceptibles de producir la muerte o alguna de las lesiones previstas en los artículos 149 y 150 de este Código, sin perjuicio de la pena que pudiera corresponder por la muerte o lesiones causadas».

\section{Estructuras temáticas conductuales de agresores sexuales en la escena del crimen}

En diversos estudios se ha analizado la estructura temática conductual de los agresores sexuales en la escena del crimen, encontrándose, en general, entre 3 y 5 temáticas diferentes. Tales temáticas no constituyen una clasificación ni una tipología, sino que deben entenderse como dimensiones: cada caso particular tendrá un determinado valor en cada una de estas dimensiones.

De esta manera, CANTER y Heritage (1990) encuentran en su estudio las dimensiones de Intimidad, Sexualidad, Violencia, Impersonal, y Criminalidad. ALISON y STEIN (2001), por su parte, encontraron tres agrupamientos de conductas, o dimensiones, en la escena del crimen, tal como fueron descritas por las víctimas, a los que denominaron Dominancia, Ganarse la confianza, y Hostilidad. En un estudio posterior de CANTER y sus colaboradores, trabajando sobre 112 casos de violación por desconocidos en Gran Bretaña, y a partir de las declaraciones de las víctimas en sede policial, se obtienen cuatro dimensiones: Hostilidad, Robo, Intimidad, y Control (CANTER, BENnel, Alison \& RedDy, 2003). Un estudio 
similar al descrito analiza 100 casos de violación cometidas por desconocidos en Finlandia (HÄKKÄNEN, LindlöF \& SANTTILA, 2004). En esta oportunidad se encontraron tres dimensiones conductuales: Hostilidad, Robo e Intimidad. También tres dimensiones aparecen en el estudio de LeHManN, Goodwill, Gallasch-Nemitz, BiedermanN, y Dahle (2013) con una muestra de 167 casos de violaciones perpetradas por desconocidos para la víctima: Explotación Sexual, Criminalidad y Hostilidad. Por último, en un reciente estudio sobre agresiones sexuales en España $(n=87)$, se han encontrado cuatro dimensiones: Hostilidad, Control, Criminalidad e Intimidad.

Parece haber un consenso de que existen cuatro grupos temáticos que abarcan la mayoría de las conductas del agresor sexual, pudiéndose asignar cada uno de estos delincuentes a uno de tales grupos, de acuerdo con su puntuación en cada una de las dimensiones.

La temática de Hostilidad estaría relacionada con la ira del agresor, éste utiliza a la víctima como vehículo para canalizar su enfado. El agresor ataca a la mujer con violencia y por sorpresa, tomándola del cuello, la tira al suelo, la arrastra hasta un lugar apartado, le rompe las ropas con sus manos o con algún elemento cortante, y la viola penetrándola vaginalmente hasta eyacular.

La temática de Control se vincularía a las fantasías sádicas del agresor. Éste intenta controlar, dominar a la víctima, tapándole la boca con las manos, tomándola del pelo, amordazándola o atándola. Una vez bajo su control procede a manosearla, y a realizarle prácticas sexuales no consentidas y acordes con sus fantasías, como penetrarla vaginal y analmente con sus dedos, o con objetos de distinto tipo. Mediante amenazas, puede exigirle a la víctima tome un rol activo en el acto sexual, desnudándose por sí misma, por ejemplo.

La temática de Criminalidad estaría relacionada con delincuentes versátiles, donde la agresión sexual sólo es otro más de sus delitos. El agresor suele pedirle a la víctima que le entregue bienes materiales tales como dinero, teléfonos móviles, etcétera. Roba, pero también satisface sus deseos sexuales con la víctima, mediante la felación, o la penetración anal. Tanto en este tema como en el de Control la víctima es tratada como un objeto por el agresor.

La temática de (pretendida) Intimidad guardaría más relación con un agresor que trata a la víctima como persona. Durante el crimen, el agresor, que tiene disminuidas sus capacidades volitivas o intelectivas, aborda a la víctima e intenta besarla, acariciarla, entablar una conversación. El sexo no consentido suele ser menos agresivo, como, por ejemplo, el cunnilingus.

En el tema de Hostilidad la motivación principal de la agresión sexual parece ser la ira, mientras que en los demás temas sería la excitación sexual. En el caso del tema de Control el sadismo surgiría a partir de la 
motivación sexual imbricada con componentes de poder y dominación sobre la persona.

\section{Características del agresor sexual y contexto del crimen}

Aunque la temática conductual que acabamos de describir ya nos brinda una información preliminar acerca de la personalidad del agresor, hay otras características del mismo que pueden ampliar mucho nuestro conocimiento acerca de su psicología y sus características demográficas. Por ejemplo, si tiene problemas psiquiátricos, o de adicción a las drogas, o si es alcohólico, o si tiene una pareja estable.

Por otro lado, el contexto en que se produce el crimen - la víctima y el ambiente- es fundamental para entenderlo. Al delito hay que analizarlo por lo menos en dos niveles, el individual y el social, para vislumbrar su mecanismo (JANOSCH GONZÁLEZ, 2013; WIKSTRÖM, 2006; WiKström, OBerWitTler, Treiber \& Hardie, 2012; WiKström, 2014). Como bien señalan MoKRos y ALISON (2002), si el mismo efecto puede deberse a diferentes causas, cualquier similitud en los delitos puede deberse a diferentes factores de contexto, enmascarando el «principio» de homología. Por otra parte, las teorías modernas de la personalidad, que deberían aplicarse en la PC, se basan en la interacción de la persona con la situación (ALISON, BENNELl, MoKros \& ORMERod, 2002).

\section{Propósito del presente estudio}

En este estudio, a partir de una muestra de agresiones sexuales $(\mathrm{n}=85)$ cometidas en España contra mujeres mayores de 12 años, analizamos variables referentes a las características del agresor sexual y al ambiente, a la conducta de la víctima, y a la posible reacción de terceros; y las vinculamos con las cuatro dimensiones de temáticas conductuales del agresor en la escena del crimen: Hostilidad, Control, Criminalidad e Intimidad.

\section{Método}

A los efectos de obtener la información básica para el estudio hemos recurrido a la base de datos del Consejo General del Poder Judicial (CENDOJ), buscando sentencias dictadas por Audiencias Provinciales por agresión sexual, del periodo de dos años comprendido entre el 1 de abril de 2015 y el 31 de marzo de 2017. Se utilizaron los siguientes criterios: 
a) El agresor debía ser de sexo masculino, actuando en solitario y desconocido para la víctima (o la víctima lo había conocido no más de 24 horas antes de la agresión sexual);

b) La víctima debía ser única y de sexo femenino, contando por lo menos con 13 años de edad en el momento del hecho; y

c) La sentencia debía ser condenatoria.

Luego de una primera lectura del contenido de las sentencias, se identificaron 10 variables nominales dicotómicas (Kerlinger \& Lee, 2000), de interés para este estudio, que se refieren a características del agresor, a conductas de la víctima, al ambiente, y a la aparición de terceras personas que, de alguna manera, cambian el curso de la agresión. Las detallamos en la tabla 1, junto con la frecuencia con la que aparecen en los 85 casos.

Tabla 1. Diez variables referentes al agresor, la víctima y al ambiente

\begin{tabular}{|c|l|c|}
\hline Variable & \multicolumn{1}{|c|}{ Descripción } & Frecuencia \\
\hline ALC & El agresor tiene problemas de alcoholismo. & $11 \%$ \\
\hline DRG & El agresor tiene problemas de adicción a las drogas. & $6 \%$ \\
\hline PSI & El agresor tiene problemas psiquiátricos. & $15 \%$ \\
\hline PAR & El agresor tiene una esposa o pareja estable. & $8 \%$ \\
\hline LPU & La agresión se cometió en un lugar público. & $48 \%$ \\
\hline LVI & $\begin{array}{l}\text { La agresión se cometió en la vivienda, portal, trabajo, } \\
\text { o coche de la víctima. }\end{array}$ & $41 \%$ \\
\hline LAG & $\begin{array}{l}\text { La agresión se cometió en la vivienda, portal, trabajo, } \\
\text { o coche del agresor. }\end{array}$ & $15 \%$ \\
\hline GRI & La víctima grita durante la agresión. & $38 \%$ \\
\hline ESC & La víctima logra escapar. & $16 \%$ \\
\hline AYU & $\begin{array}{l}\text { La policía o terceras personas acuden en ayuda de la } \\
\text { víctima. }\end{array}$ & $29 \%$ \\
\hline
\end{tabular}

Una vez determinadas las variables se le asignó a cada una de ellas, en cada uno de los 85 casos, un valor de 1 (presencia), o de 0 (ausencia).

Por otro lado, definimos nuestras temáticas de Hostilidad, Control, Criminalidad e Intimidad, basándonos en estudios anteriores (CANTER \& Heritage, 1900; Alison \& Stein, 2001; Canter, Bennel, Alison \& REdDY, 2003; HÄKKÄNEN, LindlöF \& SANTTILA, 2004; LeHMANN, GoOdWILl, Gallasch-Nemitz, BiedermanN, \& Dahle, 2013), y asignamos cada uno de 
los 85 casos a una de estas temáticas, de acuerdo con el máximo valor de sus dimensiones, una vez normalizadas, según la estructura de variables dicotómicas detallada en la tabla 2.

\section{Tabla 2. Las cuatro dimensiones de temáticas conductuales}

\section{Hostilidad}

El agresor se acerca a la víctima atacándola por sorpresa con violencia.

El agresor coge a la víctima del cuello.

$\mathrm{El}$ agresor tira al suelo a la víctima.

El agresor arrastra a la víctima.

El agresor lleva a la víctima a un lugar apartado.

$\mathrm{El}$ agresor penetra (o intenta penetrar) vaginalmente a la víctima.

El agresor eyacula durante la agresión.

\section{Control}

El agresor utiliza o muestra un arma.

El agresor amenaza de muerte (o con lastimar) a la víctima o a su familia.

El agresor amordaza, ata o venda a la víctima.

El agresor exige a la víctima que se quite la ropa, o parte de ella.

El agresor exige a la víctima que participe del acto sexual, física o verbalmente.

El agresor penetra (o intenta penetrar) analmente a la víctima.

$\mathrm{El}$ agresor introduce (o intenta introducir) un dedo en el ano de la víctima.

\section{Criminalidad}

El agresor exige a la víctima que no grite ni hable.

El agresor introduce (o intenta introducir) su miembro en la boca de la víctima.

El agresor exige a la víctima que le entregue valores. Ejemplos: dinero o un teléfono móvil.

El agresor roba valores a la víctima, distintos de fotos, prendas íntimas, etc. Ejemplos: dinero o un teléfono móvil.

\section{Intimidad}

El agresor tiene disminuidas sus capacidades volitivas o intelectivas durante la agresión.

El agresor manosea a la víctima.

El agresor besa, acaricia, se disculpa, charla, le practica cunnilingus, le roba fotos o prendas íntimas, etc. a la víctima.

$\mathrm{El}$ agresor introduce (o intenta introducir) un dedo en la vagina de la víctima.

El agresor cambia o se detiene ante la resistencia de la víctima.

Con las 10 variables de la tabla 1, y el agregado de las 4 variables que representan la asignación de cada caso a cada una de las temáticas conductuales (Hostilidad, Control, Criminalidad, Intimidad), no aplicamos el procedimiento PROXSCAL del SPSS de IBM, versión 23, 
realizando así un análisis de escalado multidimensional, o MDS (HAIR, BLACK, BABIN \& ANDERSON, 2014), utilizando la medida de distancia de Jaccard. El procedimiento MDS calcula la asociación o correlación entre todas las n variables tomadas de dos en dos. Estas asociaciones pueden ser representadas como distancias originales entre puntos en un espacio de dimensión $\mathrm{n}-1$, donde cada punto representa una de las variables. Luego, a través de un algoritmo iterativo, se logra representar los puntos en un espacio de menos dimensiones, generalmente 2 o 3, maximizando el ajuste entre la distancia original de cada par de variables, y la distancia transformada. La representación en 2 o 3 dimensiones, obviamente, facilita su interpretación. El grado de ajuste entre las distancias originales y las transformadas se mide por un valor de «estrés», de tal manera que a menor «estrés» mayor ajuste. Cuanto más cerca se encuentren dos puntos, correspondientes a dos variables, más asociadas se encuentran las mismas. Dado que las variables son dicotómicas, para medir el grado de asociación se utilizan, a partir de tablas de $2 \times 2$ (con celdas a, b, c y d), medidas de distancia como la de Lance y Williams, $(b+c) /(2 a+b+c)$, o la de Jaccard, $(b+c) /(a+b+c)$, esta última es la que hemos utilizado en el presente estudio; donde 'a' representa la presencia (valor 1) en ambas variables (1|1); ' $d$ ' la ausencia (valor 0 ) en ambas variables $(0 \mid 0)$; mientras que ' $b$ ' $y$ ' $c$ ' la presencia en una variable y la ausencia de la otra, y viceversa, respectivamente: $(1 \mid 0)$ y $(0 \mid 1)$. Hay que notar que, si los dos valores están ausentes en un caso particular, la asociación no se incrementa (al no aparecer 'd' en la fórmula), lo que es apropiado para datos provenientes de registros judiciales o policiales, donde puede ocurrir que algunas variables tengan valor de presencia, pero no se hayan registrado (HÄKKÄNEN, LINDLÖF \& SANTTILA, 2004).

Por último, se analizaron las edades de los agresores en cada una de las cuatro temáticas conductuales.

\section{Resultados}

La distribución de los 85 casos en cuanto a temáticas ha resultado de la siguiente manera: 35 casos (41\%) en Hostilidad, 12 casos (14\%) en Control, 10 casos (12\%) en Criminalidad, y 28 casos (33\%) en Intimidad.

El procedimiento MDS posterior proporcionó una solución gráfica en tres dimensiones, con una medida de Stress-1 de 0,15, en 29 iteraciones, valor que resulta aceptable para este tipo de datos. Como esperábamos, se formaron cuatro grupos de variables, cada uno encabezado por cada una de las dimensiones temáticas, ya que el valor 1 en cualquiera de ellas (asignación de un caso a la estructura) automáticamente le asignaba un valor cero a las tres restantes.

Las agrupaciones de variables se muestran en la tabla 3. 
Tabla 3. Las dimensiones conductuales y su asociación con las características del agresor, la víctima y el ambiente

\begin{tabular}{|l|l|}
\hline \multicolumn{1}{|c|}{ Dimensión Conductual } & \multicolumn{1}{c|}{ Agresor, Víctima y/o Ambiente } \\
\hline Hostilidad & LPU, GRI, AYU. \\
\hline Control & LVI, PSI. \\
\hline Criminalidad & LVI, DRG, PAR. \\
\hline Intimidad & LVI, LAG, PSI, ALC, ESC. \\
\hline
\end{tabular}

Las edades de los agresores al momento del crimen, para cada dimensión conductual, se detallan en la tabla 4.

Tabla 4. Edades del agresor en cada dimensión conductual

\begin{tabular}{|l|c|c|c|c|}
\hline $\begin{array}{c}\text { Dimensión } \\
\text { Conductual }\end{array}$ & N. ${ }^{\circ}$ de casos & Rango & Media & $\begin{array}{c}\text { Desviación } \\
\text { estándar }\end{array}$ \\
\hline Hostilidad & 30 & $19-72$ & 32,0 & 10,6 \\
\hline Control & 11 & $19-55$ & 29,7 & 9,8 \\
\hline Criminalidad & 8 & $19-42$ & 28,8 & 8,0 \\
\hline Intimidad & 23 & $19-51$ & 31,6 & 9,9 \\
\hline
\end{tabular}

\section{Discusión y conclusiones}

En este estudio, con una muestra de agresiones sexuales $(n=85)$ cometidas en España contra mujeres mayores de 12 años, analizamos variables referentes a las características del agresor sexual, al ambiente, a la víctima, y a la posible aparición en la escena de terceros; vinculándolas con las cuatro dimensiones de temáticas conductuales: Hostilidad, Control, Criminalidad e Intimidad.

En lo que concierne a la distribución de edades con respecto a las temáticas conductuales puede verse a simple vista en la tabla 4 que no hay diferencias significativas. Los valores medios oscilan entre 28,8 y 32,0 años, y las desviaciones estándar entre 8,0 y 10,6 años. La edad, en nuestra muestra, por lo tanto, no serviría de ayuda para la confección de un perfil criminológico con el objeto de discriminar sospechosos.

Por otro lado, las variables ambientales se refieren al lugar donde ocurrió la agresión. Si el crimen se cometió en la vivienda, portal, trabajo 
o coche de la víctima (LVI); o bien del agresor (LAG); o bien en un lugar público como un parque, una discoteca, una calle (LPU). En la tabla 3 puede verse que mientras que los agresores sexuales cuyas conductas en la escena del crimen coinciden con la temática de Hostilidad suelen actuar en lugares públicos (LPU), aquellos cuyas conductas coinciden con la temática de Intimidad lo hacen en lugares privados de la víctima (LVI), o en sus propios lugares privados (LAG). Aquellos cuyas conductas coinciden con la temática de Control y de Criminalidad suelen cometer la agresión en la vivienda, portal, trabajo o coche de la víctima (LVI).

El agresor sexual que es hostil hacia la víctima podría preferir los lugares públicos alejados porque en la zona privada de la mujer podría aparecer fortuitamente alguien que la ayude, o ésta podría tener más oportunidades para defenderse; y porque en la zona privada del agresor estaría exponiéndose a que sea más fácil su posterior localización y captura. O quizás el hecho de realizar el crimen en un lugar público se deba a que sale con ira, muy enfadado, a la calle a buscar a «la primera que encuentra»: sus agresiones no son premeditadas y no buscan un tipo específico de víctima (BEauRegard, 2010). Por otro lado, el hecho de cometer el delito en lugares públicos podría resultar en la aparición circunstancial de terceras personas, y es quizás por ello que la víctima tiene más tendencia a oponer resistencia gritando (GRI), como se indica en la tabla 3. De hecho, en nuestra muestra, la aparición de personas (AYU) que, de alguna manera u otra, impidieron la continuación de la agresión se concentran en el tema de Hostilidad, donde, como ya hemos comentado, el agresor parece actuar violentamente a partir de la ira, del enfado, del menosprecio hacia la mujer en general, quizás por sentirse incapaz de lograr una verdadera relación de intimidad, o por mantener creencias equivocadas, errores cognitivos, acerca de las mujeres, tal vez por haber sido educado, o continuar viviendo, en un ambiente de tipo "machista», donde se menosprecia y se degrada a la mujer. Por otro lado, parece haber una relación entre el grado de hostilidad o violencia y el lugar donde ocurre la agresión: quienes actúan en lugares públicos suelen aplicar mayor violencia, de acuerdo con Hewitt y BEAUREGARD (2014), lo que también concuerda con nuestros hallazgos. Teniendo en cuenta, además, que habría una relación entre la fuerza física aplicada por el agresor con respecto al lugar, privado o público de la agresión: si no hubo fuerza física hay mayor posibilidad de que el delito se haya cometido en un lugar privado, ya sea de la víctima (LVI), o del agresor (LAG) (BALEMBa y BEAUREGARD, 2013).

Con respecto al tema de Control, encontramos aquí a personas con conductas sexuales desviadas, de índole sádica, y el hecho que actúen con preferencia en la zona privada de la víctima, al menos como se deduce de nuestra muestra, podría deberse a su deseo de poder interactuar el mayor tiempo posible con la mujer, una vez controlada, atada, amordazada o vendada. Como así también la posibilidad de poder desplegar con total tranquilidad toda una serie de elementos necesarios para sus 
no consentidos fines, como podrían ser bridas, sogas cuchillos, etcétera. Todo esto es compatible, como se ve en la tabla 3, con que esta clase de sujetos tiendan a tener problemas psiquiátricos (PSI). El agresor sexual sádico suele planear cuidadosamente sus agresiones. Sus fantasías sexuales desviadas influencian tanto el lugar que elige para agredir como también el tipo de víctima (BEAUREGARD, 2010).

Los individuos cuyas conductas en la escena del crimen coinciden con la temática de Criminalidad también muestran una preferencia por la zona privada de la víctima. Esto podría explicarse por sus variados objetivos, principalmente robar bienes materiales y agredir sexualmente. Estos sujetos, que podrían poseer una personalidad antisocial, o psicopática, que buscan el placer inmediato, carecen de empatía, y que no se preocupan por los demás, a quienes tratan únicamente como objetos necesarios para sus fines (BEAUREGARD, 2010); podrían tener, cuando no están delinquiendo, una vida social de aparente normalidad, integrados en la sociedad, e incluso manteniendo una relación de pareja estable (PAR). Por otro lado, su búsqueda de placer inmediato podría llevarlos a tener problemas de adicción a las drogas (DRG), como también se ve en este estudio (ver tabla 3).

Por último, los sujetos que responden a la temática de Intimidad aparecen como los menos agresivos en nuestra muestra, y de hecho es precisamente en esta temática donde se concentrar aquellos casos donde la víctima escapa del agresor (ESC). Contrariamente a quienes actúan, como acabamos de ver, en la zona privada de la víctima por cuestiones de control o criminalidad, los agresores que buscan una pretendida intimidad lo harían porque no pueden medir las consecuencias de sus actos, o creen que lograrán, en definitiva, que la víctima acceda a sus requerimientos. Tal fallo en las habilidades sociales se ve acrecentado al estar generalmente disminuidas en estos casos las capacidades volitivas o intelectivas del agresor. Dos características asociadas a esta temática son los problemas psiquiátricos (PSI) o los problemas con el alcohol (ALC).

En definitiva, creemos que estos resultados, con respecto a algunas características del agresor, el lugar donde se produce la agresión, y las conductas de las víctimas o de terceras personas, podrían ser de utilidad a la hora de confeccionar un perfil criminológico para priorizar sospechosos.

Una de las limitaciones de nuestro estudio es que refleja las conductas y características de agresores sexuales que fueron identificados y condenados. No sabemos hasta qué punto podrían ser diferentes las conductas de aquellos agresores sexuales que no fueron condenados o que no fueron identificados. Otra de las limitaciones del estudio es que la información proviene de sentencias judiciales, cuya finalidad no es la de proporcionar datos en el marco de una investigación científica (pero véase, por ejemplo, ALISON, SNOOK \& STEIN; 2001). 
Recordemos que los delitos no se producen sólo a partir de la propensión a cometerlos por parte del autor, también el ambiente juega un papel fundamental: ¿cuáles son las características físicas y psicosociales del ambiente? De acuerdo con Smallbone y Cale (2016), muchas de las teorías sobre la agresión sexual, aunque importantes en un contexto clínico, se centran en la prevención y tratamiento de la agresión sexual, en el individuo y en causas distales, pero no en aspectos situacionales. Por nuestra parte, interesados sobre todo en la aplicación práctica de los perfiles criminológicos, deberemos considerar tanto las variables individuales como las ambientales, y las causas proximales además de las distales. Sólo en la situación, definida como la interacción del agresor con el ambiente (incluida la víctima en este último), puede comprenderse cabalmente el mecanismo de la agresión sexual. Además de preguntarnos por las normas morales y el autocontrol del individuo, debemos preguntarnos también por la «moral del ambiente», y las medidas de control que posee o no (vigilancia, cámaras de seguridad, etcétera). Por supuesto, sin olvidar que nunca las conductas de la víctima, ni el ambiente en el que ésta se encuentra, la responsabiliza en lo más mínimo de la agresión sexual: siempre toda la responsabilidad del delito recae única y exclusivamente sobre el agresor.

A partir de temáticas conductuales del agresor sexual en la escena del crimen, hemos encontrado algunas asociaciones con variables referidas a las características del agresor, la víctima y el ambiente, conocimientos que ayudarían en la elaboración de perfiles psicológicos hipotéticos del agresor, en casos futuros. La interacción del agresor con víctima y ambiente se muestra crucial, y esperamos que los futuros estudios se centren más en el evento criminal en su totalidad, y no exclusivamente en las conductas del agresor en la escena del crimen.

Los autores no reconocen ningún conflicto de intereses.

\section{Bibliografía}

Alison, L. J., Bennell, C., Mokros, A., \& Ormerod, D. (2002). The personality paradox in offender profiling: A theoretical review of the processes involved in deriving background characteristics from crime scene actions. Psychology, Public Policy, and Law, 8, 115-135.

Alison, L. J., SNOOK, B., \& STEIN, K. L. (2001). Unobtrusive measurement: using police information for forensic research. Qualitative Research, 1(2), 241-254.

Alison, L. J., \& STEIN, K. (2001). Vicious circles: How accounts of stranger sexual assault reflect abusive variants of conventional interactions. The Journal of Forensic Psychiatry, 12, 515-538. 
Balemba, S., \& Beauregard, E. (2013). Where and when? Examining spatiotemporal aspects of sexual assault events. Journal of sexual aggression, 19(2), 171-190.

BEAURegard, E. (2010), Rape and sexual assault in investigative psychology: the contribution of sex offenders' research to offender profiling. J. Investig. Psych. Offender Profil., 7: 1-13. doi:10.1002/jip. 114

Canter, D. V., Bennell, C., Alison, L. J., \& Reddy, S. (2003). Differentiating sex offences: A behaviorally based thematic classification of stranger rapes. Behavioral Sciences and the Law, 21, 157-174.

Canter, D. V., \& Heritage, R. (1990). A multivariate model of sexual offence behaviour: Developments in 'offender profiling'. The Journal of Forensic Psychiatry, 1(2), 185-212.

CANTER, D. V., \& Youngs, D. (2009). Investigative psychology: Offender profiling and the analysis of criminal action. West Sussex, UK: Wiley.

Douglas, J. E., Ressler, R. K., Burgess, A. W., \& Hartman. C. R. (1986). Criminal profiling from crime scene analysis. Behavioral Sciences \& the Law, 4, 401-421.

Goodwill, A. M., Lehmann, R. J. B., Beauregard, E., \& Andrei, A. (2016). An action phase approach to offender profiling. Leg Crim Psychol, 21: 229-250.

Hair, J.F., Black, W.C., Babin, B.J., \& ANDERson, R.E. (2014). Multivariate Data Analysis. Harlow: England: PEARSON.

HÄKKäNen, H., LindlöF, P., \& SAnttila, P. (2004). Crime scene actions and offender characteristics in a sample of Finnish stranger rapes. Journal of Investigative Psychology and Offender Profiling, $1,17-32$.

Hewitt, A., \& Beauregard, E. (2014) Sexual Crime and Place: The Impact of the Environmental Context on Sexual Assault Outcomes. Journal of Criminal Justice 42(5), pp 375-383.

Hicks, S. J., \& SALES, B. D. (2009). Criminal profiling. Milano, Italia: Raffaello Cortina Editore.

JANOSCH GoNZÁLEZ, H. (2013). Fundamentos filosóficos de la criminología en Hirschi y Wikström. ¿Popper o Bunge? En A. SERrano Maíllo, A. y Ch. BIRKBeck (Eds.) La Generalidad de la Teoría del Autocontrol. Una primera extensión de la teoría general del delito a los países de habla hispana. Madrid, España: Editorial Dykinson.

KERLINGER, F.N., \& LEE, H.B. (2000). Foundations of behavioral research. New York: Harcourt College Publishers.

Konvalina-Simas, T., Turvey, B.E., \& Kenndedy, D.B. (2016). Criminologia Forense. Lisboa, Portugal: Letras e Conceitos Lda. 
Lehmann, R. J. B., Goodwill, A. M., Gallasch-Nemitz, F., Biedermann, J., \& DAHLE, K.-P. (2013). Applying crime scene analysis to the prediction of sexual recidivism in stranger rapes. Law and Human Behavior, 37(4), 241-254.

Mokros, A., \& ALISON, L. J. (2002). Is offender profiling possible? Testing the predicted homology of crime scene actions and background characteristics in a sample of rapists. Legal and Criminological Psycholo$g y, 7(1), 25-43$.

Smallbone, S., \& Cale, J. (2016). Situational theories. In D.P. BOEr (Ed.) The Wiley Handbook on the Theories, Assessment, \& Treatment of Sexual Offending. Volume I: Theories, edited by A. Beech and T. Ward. West Sussex, UK: Wiley.

Soto Castro, J.E. (2017). Manual de investigación psicológica del delito, 2. ${ }^{a}$ edición. Madrid, España: Pirámide.

Soto Castro, J.E., González Ordi, H., \& Pérez Nieto, M.A. (2014). La investigación psicológica de los delitos violentos. Psicopatología Clínica, Legal y Forense, Vol. 14.

TuRveY, B.E. (Ed.) (2012). Criminal profiling: An introduction to behavioral evidence analysis. London, UK: Elsevier.

WiKstRÖM, P-O.H. (2006). Individuals, settings, and acts of crime: situational mechanisms and the explanation of crime. In P-O.H. Wikström \& R.J. Sampson (Eds.) The explanation of crime: context, mechanisms, and development. Cambridge, UK: Cambridge University Press.

Wikström, P-O.H., Oberwittler, D., Treiber, K. \& Hardie, B. (2012). Breaking Rules: The Social and Situational Dynamics of Young People's Urban Crime. Oxford, UK: Oxford University Press.

Wikström, P-O.H. (2014). Why crime happens: A situational action theory. In G. Manzo (Ed.) Analytical Sociology: Actions and Networks. West Sussex, UK: Wiley. 
